Radial and Nonradial Pulsations as Probes of Stellar Physics ASP Conference Series, Vol. 259, 2002

C. Aerts, T.R. Bedding, \& J. Christensen-Dalsgaard, eds.

\title{
P-Mode Observations on $\alpha$ Cen A with CORALIE
}

F. Carrier, F. Bouchy, G. Meynet, A. Maeder

Observatoire de Genève, CH-1290 Sauverny, Switzerland

J. Provost, G. Berthomieu, P. Morel, F. Thévenin

Département Cassini, UMR CNRS 6529, Observatoire de la Côte d'Azur, BP 4229, 06304 Nice CEDEX 4, France

Abstract. We have made a clear detection of p-mode oscillations in the G2V star $\alpha$ Cen A with the CORALIE spectrograph. The power spectrum clearly shows several identifiable peaks between 1.8 and $2.9 \mathrm{mHz}$. A preliminary astrophysical interpretation of these p-modes is presented.

\section{Observations}

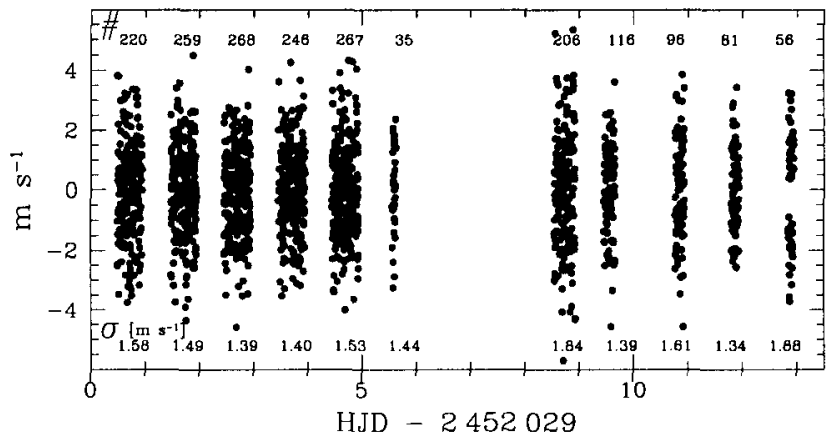

Figure 1. Radial velocity measurements of $\alpha$ Cen A. A 3 order polynomial fit subtraction was applied on each night. The dispersion reaches $1.53 \mathrm{~m} \mathrm{~s}^{-1}$. The number of Doppler measurements and their dispersion are indicated for each night.

$\alpha$ Cen A was observed over 13 nights in May 2001 with the CORALIE echelle spectrograph (Carrier et al., these proceedings) mounted on the 1.2$m$ Swiss telescope at the ESO La Silla Observatory. Observations and data reduction are described in Bouchy \& Carrier (2001). In total, 1850 spectra were collected with typical signal-to-noise ratio in the range $300-420$ at $550 \mathrm{~nm}$. The radial velocities are shown in Fig. 1. 


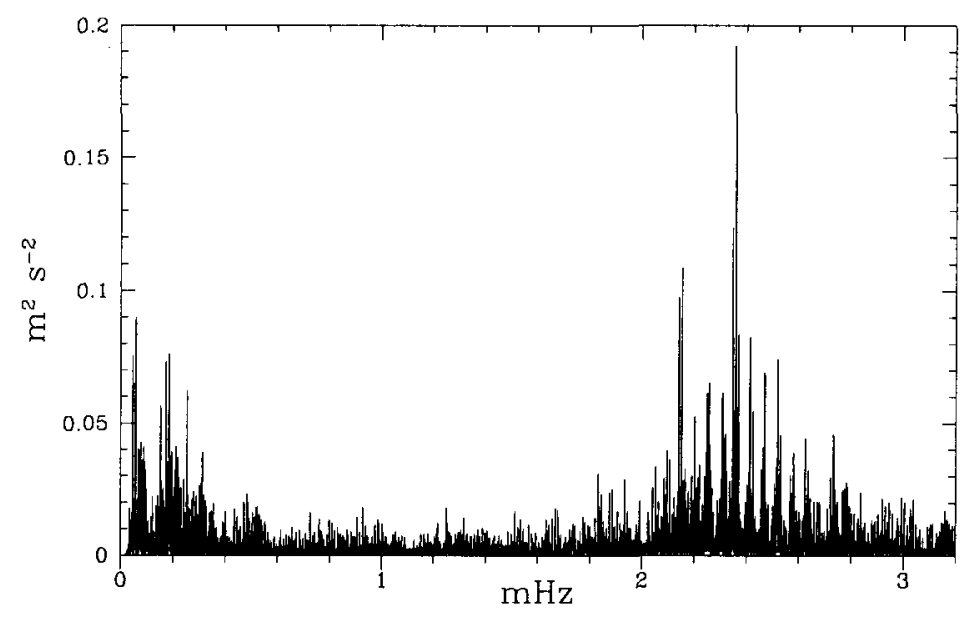

Figure 2. Power spectrum of the radial velocity measurements of $\alpha$ Cen $\mathrm{A}$. The mean white noise level in the range $0.6-1.5 \mathrm{mHz}$ reaches $0.00239 \mathrm{~m}^{2} \mathrm{~s}^{-2}$, namely $4.3 \mathrm{~cm} \mathrm{~s}^{-1}$ in amplitude. This noise level corresponds to a velocity accuracy of $1.05 \mathrm{~ms}^{-1}$. The mean peak amplitude is in the range $29-33 \mathrm{~cm} \mathrm{~s}^{-1}$.

\section{P-mode characterization}

The power spectrum of the velocity time series is shown in Fig. 2 and exhibits a series of peaks between 1.8 and $2.9 \mathrm{mHz}$ modulated by a broad envelope. Toward the lowest frequencies $(\nu<0.6 \mathrm{mHz})$, the power rises and scales inversely with frequency squared as expected for instrumental instabilities. The photon noise uncertainty of our measurements is estimated to $0.70 \mathrm{~m} \mathrm{~s}^{-1}$, whereas the velocity accuracy corresponds to $1.05 \mathrm{~m} \mathrm{~s}^{-1}$. The strongest modes are identified in Fig. 3

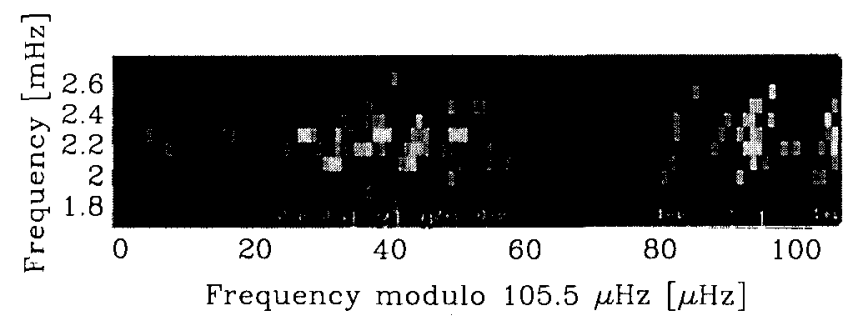

Figure 3. Echelle diagram of $\alpha$ Cen A oscillations. $\nu$ is $11.57 \mu \mathrm{Hz}$.

and listed in Table 1. Our previous identification in Bouchy \& Carrier (2001) based on the 5 first nights did not distinguish between the modes $\ell=0$ and $\ell=2$. We deduce from Table 1 the average large $\langle\Delta \nu\rangle$ and small $\left\langle\delta \nu_{02}\right\rangle$ splittings, respectively $105.5 \pm 0.3$ and $6.6 \pm 0.4 \mu \mathrm{Hz}$. 
Table 1. Mode frequencies (in $\mu \mathrm{Hz}$ ). The frequency resolution of the time series is $0.9 \mu \mathrm{Hz}$.

\begin{tabular}{l|ccc}
\hline & $\ell=0$ & $\ell=1$ & $\ell=2$ \\
\hline$n=15$ & & & 1833.0 \\
$n=16$ & 1841.2 & 1887.2 & 1935.0 \\
$n=17$ & & 1991.9 & 2041.5 \\
$n=18$ & & 2095.6 & 2145.9 \\
$n=19$ & 2152.8 & 2202.8 & 2251.3 \\
$n=20$ & 2258.3 & 2308.9 & 2358.3 \\
$n=21$ & 2364.2 & 2414.2 & 2464.1 \\
$n=22$ & 2470.0 & 2519.3 & 2568.5 \\
$n=23$ & 2573.2 & 2625.5 & 2672.2 \\
$n=24$ & 2679.7 & 2733.0 & 2782.9 \\
$n=25$ & & 2837.4 & \\
\hline
\end{tabular}

\section{Astrophysical interpretation : comparison with models}

\subsection{Stellar evolution models of Nice}
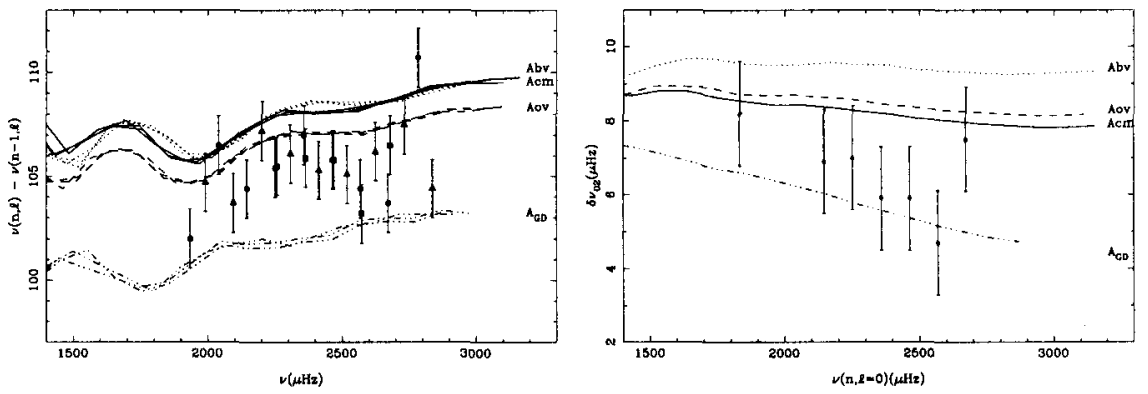

Figure 4. Left: Variations of the large spacing between modes of consecutive radial order for $p$-modes of degree $\ell=0$ (round), $\ell=1$ (triangle) and $l=2$ (square) versus frequency. Right: Variations of the small frequency spacing $\delta \nu_{02}=\nu_{n, 0}-\nu_{n-1,2}$ versus frequency.

The variation versus frequency of the large and small spacings obtained from our observations are compared in Fig. 4 with the values deduced from the models developed by Morel et al. (2000). The observational constraints of the calibration and the properties of the calibrated models are given in Table 2. Our observations suggest for $\alpha$ Cen A a model with properties intermediate between the two sets of models, i.e. a mass between 1.10 and $1.16 \mathrm{M}_{\odot}$. New calibrations of the $\alpha$ Cen binary system are in progress, with different physics in order to better interpret the observed oscillations.

\subsection{Stellar evolution models of Geneva}

The stellar evolution code of Geneva was processed with initial chemical abundances fixed to: $\mathrm{X}_{i}=0.675$ and $\mathrm{Y}_{i}=0.297$, corresponding to twice the solar 
Table 2. Upper panel: Observational constraints adopted for the calibration of models. Lower panel: Global characteristics derived from the calibrated models.

\begin{tabular}{|c|c|c|c|c|}
\hline \multirow{6}{*}{$\begin{array}{l}\text { Models } \\
M / M_{\odot} \\
T_{\text {eff }}[K] \\
\log g \\
{\left[\frac{\mathrm{Fe}}{\mathrm{H}}\right]_{\mathrm{i}}} \\
L / L_{\odot} \\
\end{array}$} & \multicolumn{2}{|c|}{$A_{B V}, A_{o v} \& A_{C M}$} & \multicolumn{2}{|r|}{$\bar{A}_{G D}$} \\
\hline & \multirow{3}{*}{\multicolumn{2}{|c|}{$\begin{array}{c}1.16 \pm 0.031 \\
5790 \pm 30 \\
4.32 \pm 0.05\end{array}$}} & 1.10 & \\
\hline & & & \multirow{2}{*}{\multicolumn{2}{|c|}{$\begin{array}{l}5770 \pm 50 \\
4.28 \pm 0.02\end{array}$}} \\
\hline & & & & \\
\hline & \multirow{2}{*}{\multicolumn{2}{|c|}{$\begin{array}{c}0.20 \pm 0.02 \\
1.534 \pm 0.103\end{array}$}} & \multirow{2}{*}{\multicolumn{2}{|c|}{$\begin{array}{c}0.22 \pm 0.02 \\
1.572 \pm 0.135\end{array}$}} \\
\hline & & & & \\
\hline Models & $\mathrm{A}_{\mathrm{BV}}$ & $\bar{A}_{O V}$ & $A_{C M}$ & $A_{G D}$ \\
\hline$t \mid M y r]$ & 2710 & 3530 & 4086 & 5640 \\
\hline & 0.284 & 0.279 & 0.271 & 0.300 \\
\hline$\left(\frac{Z}{X}\right)_{i}$ & 0.0443 & 0.0450 & 0.0450 & 0.0480 \\
\hline & 1.53 & 1.64 & 0.96 & 1.86 \\
\hline
\end{tabular}
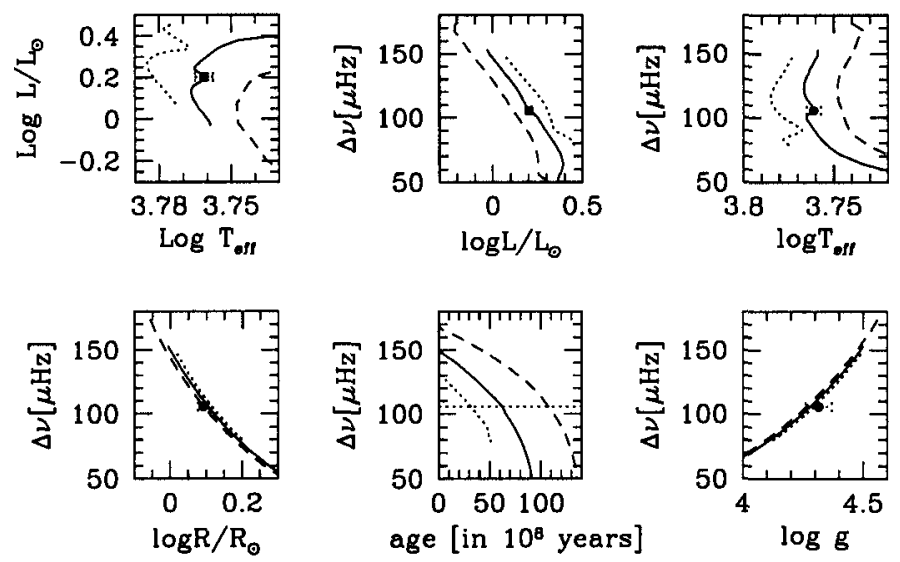

Figure 5. The dashed, solid and dotted lines correspond respectively to the evolutionary track of stars with a mass of $1.0,1.1$ and $1.2 \mathrm{M}_{\odot}$. The point represents the parameters deduced from observations.

$\mathrm{Z}_{i} / \mathrm{X}_{i}$ value. Fig. 5 shows the evolutionary tracks of stars with $1.0,1.1$ and $1.2 \mathrm{M}_{\odot}$ in the H-R diagram and in diagrams of the observed large splitting versus stellar parameters. These results suggest a mass near $1.1 \mathrm{M}_{\odot}$ for $\alpha$ Cen $\mathrm{A}$.

\section{References}

Bouchy, F. \& Carrier, F., 2001, A\&A, 374, L5

Morel, P., Provost, J., Lebreton, Y., et al. 2000, A\&A, 363, 675 